\title{
The Mellin transform method as an alternative analytic solution for the valuation of geometric Asian option
}

\author{
Fadugba Sunday Emmanuel \\ Department of Mathematical Sciences, Ekiti State University, Ado Ekiti, Nigeria \\ Email address: \\ emmasfad2006@yahoo.com,sunday.fadugba@eksu.edu.ng
}

To cite this article:

Fadugba Sunday Emmanuel. The Mellin Transform Method as an Alternative Analytic Solution for the Valuation of Geometric Asian Option. Applied and Computational Mathematics. Special Issue: Computational Finance. Vol. 3, No. 61, 2014, pp. 1-7. doi: 10.11648/j.acm.s.2014030601.11

\begin{abstract}
This paper presents the Mellin transform method as an alternative analytic solution for the valuation of geometric Asian option. Asian options are options in which the variable is the average price over a period of time. The analytical solution of the Black-Scholes partial differential equation for Asian option is known as an explicit formula, this is due to the fact that the geometric average of a set of lognormal random variables is lognormally distributed. We derive a closed form solution for a continuous geometric Asian option by means of the partial differential equations. We also provide an alternative method for solving geometric Asian options partial differential equations using the Mellin transform method.
\end{abstract}

Keywords: Analytic Solution, Asian Option, Black-Scholes Model, Mellin Transform Method, Partial Differential Equation

\section{Introduction}

Numerical methods are needed for pricing options in cases where analytic solutions are either unavailable or not easily computable. Examples of the former include American style options and most discretely observed path dependent options, while the latter includes the analytic formula for valuing continuously observed Asian options which is very difficult to calculate for a wide range of parameter values encountered in practice.

The subject of numerical methods in the area of options valuation and hedging is very broad. A wide range of different types of contracts are available and in many cases there are several candidate models for the stochastic evolution of the underlying state variables.

Asian options are less sensitive to market fluctuations near the expiry date. However these options have proved to be much more difficult to price than other options.

There are many techniques developed in the literature to price Asian options. H. Geman and M. Yor [4] used a Laplace transform to price Asian option. They also exploit the relationship between the Geometric Brownian motion and the Bessel process with a stochastic time change and the additive property of the Bessel process. Rogers L. and
Shi Z. [10] reduced the partial differential equation for Asian option to the partial differential equation in two variables instead three and then, they used numerical procedure to solve it. They also derived lower-bound formulas for Asian options by computing the expectation based on some zero-mean Gaussian variable. Zieneb A.E. and Rokiah R.A. [16] derived analytical solution for an arithmetic Asian option using Mellin transforms. They derived a closed form solution for a continuous arithmetic Asian option by means of partial differential equations. Monte Carlo method for pricing some path dependent options was considered by Nwozo C.R. and Fadugba S.E. [6]. Some Numerical Methods for Options Valuation was also considered by Nwozo C.R. and Fadugba S.E. [7]. They considered three special numerical methods in finance for the valuation of both vanilla and exotic options. Performance measure of Laplace transforms for pricing path dependent options was considered by Nwozo C.R. and Fadugba S.E. [8]. The competitive Monte Carlo method for the pricing of Asian options was considered by Lapeyre B. and Temam E. [5].

For a survey of different numerical methods and their 
performances in derivatives pricing see ([1], [2], [3], [5], [9], [11], [12], [13], [14]) just to mention a few

In this paper, we consider the Mellin transform method as an alternative analytical solution for the valuation of continuous geometric Asian option.

\section{Asian Options}

Path dependent options are contingent claims whose values depend on the sequence of prices of the underlying asset during the whole or part of the option's life rather than just the final price of the asset. Examples are Asian, Barrier and lookback options.

Asian or Average options are options whose payoff depends on the average price of the underlying asset during at least some part of the life of the option. They are also contingent claims in which the underlying variable is the average price over a period of time. Because of this fact, Asian options have a lower volatility and hence rendering them cheaper relative to their European counterparts. They are commonly traded on currencies and commodity products which have low trading volumes. They were originally used in 1987 when Banker's Trust Tokyo office used them for pricing average options on crude oil contracts and hence the name "Asian" option.

There are different types of Asian options namely:

- Continuous arithmetic average Asian option

The Call and put options are given by

$$
f=\max \left(\frac{1}{T} \int_{0}^{T} S_{t} d t-K\right)
$$

and

$$
f=\max \left(K-\frac{1}{T} \int_{0}^{T} S_{t} d t\right)
$$

respectively.

- Continuous geometric average Asian option

The Call and put options are given by

$$
f=\max \left(e^{\frac{1}{T} \int_{0}^{T} \log S_{t} d t}-K\right)
$$

and

$$
f=\max \left(K-e^{\frac{1}{T} \int_{0}^{T} \log S_{t} d t}\right)
$$

respectively.

- Discrete arithmetic average Asian option

The call and put options are given by

$$
f=\max \left(\frac{1}{n+1} \sum_{i=0}^{n} S\left(\frac{i T}{n}\right)-K\right)
$$

and

$$
f=\max \left(K-\frac{1}{n+1} \sum_{i=0}^{m} S\left(\frac{i T}{n}\right)\right)
$$

- Discrete geometric average Asian option The call and put options are given by

$$
f=\max \left(e^{\left(\frac{1}{n+1} \sum_{i=0}^{n} \log S\left(\frac{i T}{n}\right)\right)}-K\right)
$$

and

$$
f=\max \left(K-e^{\left(\frac{1}{n+1} \sum_{i=0}^{n} \log S\left(\frac{i T}{n}\right)\right)}\right)
$$

Denote the price of the arithmetic average Asian call and put options at time $0 C_{a, K}\left(S_{0}, T\right)$ and $P_{a, K}\left(S_{0}, T\right)$. Also the price of the geometric Asian call and put options at time $0 \quad$ are denoted by $C_{g, K}\left(S_{0}, T\right)$ and $P_{g, K}\left(S_{0}, T\right)$ respectively.

Proposition 2.0

The following inequalities hold in the discrete case

$$
\left.\begin{array}{l}
C_{g, K}\left(S_{0}, T\right) \leq C_{a, K}\left(S_{0}, T\right) \\
P_{g, K}\left(S_{0}, T\right) \geq P_{a, K}\left(S_{0}, T\right)
\end{array}\right\}
$$

Proof:

This follows from the inequality between the geometric and arithmetic means

$$
\left(\prod_{i=0}^{n} S\left(\frac{i T}{n}\right)\right)^{\frac{1}{n+1}} \leq \frac{1}{n+1} \sum_{i=0}^{n} S\left(\frac{i T}{n}\right)
$$

together with the fact that $\max \left(S_{t}-K, 0\right)$ is increasing in $S_{t}$ while $\max \left(K-S_{t}, 0\right)$ is decreasing in $S_{t}$

\subsection{Closed Form Solution for the Geometric Average Asian Options}

We make the following assumptions that

- The price of the underlying asset follows a lognormal distribution continuous in time

- The product of log-normal distributed random 
variables is also log-normal distributed

- The sum of log-normal distributed random variables is not log-normal distributed

With the above assumption we expect that the pricing of geometric average Asian options should be easy to deal with, while for arithmetic average ones it may be relatively more complicated to handle. In fact, the pricing formula of geometric average Asian options can be derived in the Black-Scholes framework.

The payoff function for the discrete geometric average call option is given by

$$
\left.\begin{array}{rl}
f & =\max \left(e^{\left(\frac{1}{n+1} \sum_{i=0}^{n} \log S\left(\frac{i T}{n}\right)\right)}-K\right) \\
& =\max \left(\left(\prod_{i=0}^{n} S\left(\frac{i T}{n}\right)\right)^{\frac{1}{n+1}}-K\right)
\end{array}\right\}
$$

But

$$
\left.\begin{array}{rl}
\prod_{i=0}^{n} S_{t_{i}}= & \left(\frac{S_{t_{n}}}{S_{t_{n-1}}}\right)\left(\frac{S_{t_{n-1}}}{S_{t_{n-2}}}\right)^{2}\left(\frac{S_{t_{n-2}}}{S_{t_{n-3}}}\right)^{3}\left(\frac{S_{t_{n-3}}}{S_{t_{n-4}}}\right)^{4} \cdots \\
& \left(\frac{S_{t_{4}}}{S_{t_{3}}}\right)^{n-3}\left(\frac{S_{t_{3}}}{S_{t_{2}}}\right)^{n-2}\left(\frac{S_{t_{2}}}{S_{t_{1}}}\right)^{n-1}\left(\frac{S_{t_{1}}}{S_{t_{0}}}\right)^{n} S_{0}^{n+1}
\end{array}\right\}
$$

Where

$$
\begin{aligned}
\ln \left(\frac{\left(\prod_{i=0}^{n} S_{t_{i}}\right)^{\frac{1}{n+1}}}{S_{0}}\right) & =\frac{1}{n+1} \ln \left(\frac{\left(\left(\frac{S_{t_{n}}}{S_{t_{n-1}}}\right)\left(\frac{S_{t_{n-1}}}{S_{t_{n-2}}}\right)^{2}\left(\frac{S_{t_{n-2}}}{S_{t_{n-3}}}\right)^{3}\left(\frac{S_{t_{n-3}}}{S_{t_{n-4}}}\right)^{4} \ldots\left(\frac{S_{t_{2}}}{S_{t_{1}}}\right)^{n-3}\left(\frac{S_{t_{3}}}{S_{t_{2}}}\right)^{n-2}\left(\frac{S_{t_{2}}}{S_{t_{1}}}\right)^{n-1}\left(\frac{S_{t_{1}}}{S_{t_{0}}}\right)^{n} S_{0}^{n+1}\right)}{S_{0}^{n+1}}\right) \\
& \left.=\frac{1}{n+1} \ln \left(\left(\frac{S_{t_{n}}}{S_{t_{n-1}}}\right)\left(\frac{S_{t_{n-1}}}{S_{t_{n-2}}}\right)^{2}\left(\frac{S_{t_{n-2}}}{S_{t_{n-3}}}\right)^{3}\left(\frac{S_{t_{n-3}}}{S_{t_{n-4}}}\right)^{4} \ldots\left(\frac{S_{t_{2}}}{S_{t_{1}}}\right)^{n-3}\left(\frac{S_{t_{3}}}{S_{t_{2}}}\right)^{n-2}\left(\frac{S_{t_{2}}}{S_{t_{1}}}\right)^{n-1}\left(\frac{S_{t_{1}}}{S_{t_{0}}}\right)^{n}\right)^{n}\right)
\end{aligned}
$$

Substituting (13) into (15) we have

$$
\left.\begin{array}{rl}
\ln \left(\frac{\left(\prod_{i=0}^{n} S_{t_{i}}\right)^{\frac{1}{n+1}}}{S_{0}}\right) & =\frac{1}{n+1}\left((1+2+3+4+\ldots+n)\left(\left(r-\frac{\sigma^{2}}{2}\right) \frac{T}{n}+\sigma \sqrt{\left(\frac{T}{n}\right)} X_{1}+X_{2}+X_{3}+X_{4}+\ldots+X_{n}\right)\right) \\
& =\left(\frac{2 r-\sigma^{2}}{2}\right) T+\left(\frac{\sigma \sqrt{\left(\frac{T}{n}\right)} X_{1}+X_{2}+X_{3}+X_{4}+\ldots+X_{n}}{n+1}\right)
\end{array}\right\}
$$


Using the properties of independent normal random variables i.e. additive mean and variance, we have that

$$
\left(\frac{\sigma \sqrt{\left(\frac{T}{n}\right)} X_{1}+X_{2}+X_{3}+X_{4}+\ldots+X_{n}}{n+1}\right) \sim N\left(0, \frac{(n+0.5) \sigma^{2} T}{3(n+1)}\right)=\sqrt{\frac{(n+0.5) \sigma^{2} T}{3(n+1)}} Z
$$

Substituting (17) into (16) we have

$$
\begin{aligned}
\ln \left(\frac{\left(\prod_{i=0}^{n} S_{t_{i}}\right)^{\frac{1}{n+1}}}{S_{0}}\right) & =\left(\frac{2 r-\sigma^{2}}{2}\right) T+\left(\sqrt{\frac{(n+0.5) \sigma^{2} T}{3(n+1)} Z}\right) \\
& =\left(\frac{2 r-\sigma^{2}}{4}\right)+\frac{1}{2}\left(\frac{(n+0.5) \sigma^{2}}{3(n+1)}\right)-\frac{1}{2}\left(\frac{(n+0.5) \sigma^{2}}{3(n+1)}\right)+\left(\sqrt{\frac{(n+0.5) \sigma^{2} T}{3(n+1)}} Z\right)
\end{aligned}
$$

Setting

$$
\left.\begin{array}{l}
\sigma_{0}=\sqrt{\frac{(n+0.5) \sigma^{2}}{3(n+1)}} \\
\rho=\frac{1}{2}\left(\left(\frac{2 r-\sigma^{2}}{2}\right)+\left(\frac{(n+0.5) \sigma^{2}}{3(n+1)}\right)\right)=\frac{1}{2}\left(\frac{2 r-\sigma^{2}}{2}+\sigma_{0}^{2}\right)
\end{array}\right\}
$$

Hence

$$
\ln \left(\frac{\left(\prod_{i=0}^{n} S_{t_{i}}\right)^{\frac{1}{n+1}}}{S_{0}}\right)=\left(\rho-\frac{\sigma_{0}^{2}}{2}\right) T+\sigma_{0} \sqrt{T} Z
$$

From (20), we have that

$$
\left(\prod_{i=0}^{n} S_{t_{i}}\right)^{\frac{1}{n+1}}=S_{0} e^{\left(\left(\rho-\frac{\sigma_{0}^{2}}{2}\right) T+\sigma_{0} \sqrt{T} Z\right)}
$$

Using the risk-neutral method, the price of the geometric average Asian call option is given by

$$
\left.\begin{array}{c}
C_{g, k}\left(S_{0}, T\right)=e^{-r T} E\left(\max \left(\left(\prod_{i=0}^{n} S\left(\frac{i T}{n}\right)\right)^{\frac{1}{n+1}}-K\right)\right) \\
=e^{(-r T+\rho T-\rho T)} E\left(\max \left(\left(\prod_{i=0}^{n} S_{t_{i}}\right)^{\frac{1}{n+1}}-K\right)\right)
\end{array}\right\}
$$

$$
\left.\begin{array}{rl}
C_{g, k}\left(S_{0}, T\right) & \left.=e^{((\rho-r) T-\rho T)} E\left(\max \left(S_{0} e^{\left(\left(\rho-\frac{\sigma_{0}^{2}}{2}\right) T+\sigma_{0} \sqrt{T} Z\right)}-K\right)\right)\right\} \\
& =e^{(\rho-r) T} C_{K}\left(S_{0}, T\right)
\end{array}\right\}
$$

Where the price of a European call option with volatility $\sigma_{0}$ and the risk-neutral interest rate $\rho$ is given below from (23)

$$
\left.C_{k}\left(S_{0}, T\right)=e^{-\rho T} E\left(\max \left(S_{0} e^{\left(\left(\rho-\frac{\sigma_{0}^{2}}{2}\right) T+\sigma_{0} \sqrt{T} Z\right.}\right)-K\right)\right)
$$

Using analytical pricing formula, we have that

$$
C_{g, k}\left(S_{0}, T\right)=e^{(\rho-r) T}\left(S_{0} N\left(d_{1}\right)-K e^{-\rho T} N\left(d_{2}\right)\right)
$$

Where

$$
\left.\begin{array}{l}
d_{1}=\left(\frac{\ln S_{0}-\ln K+\left(\rho+0.5 \sigma_{0}^{2}\right) T}{\sigma \sqrt{T}}\right) \\
d_{2}=\left(\frac{\ln S_{0}-\ln K+\left(\rho-0.5 \sigma_{0}^{2}\right) T}{\sigma \sqrt{T}}\right) \\
N(x)=\frac{1}{2 \pi} \int_{-\infty}^{x} e^{-\frac{u^{2}}{2}} d u
\end{array}\right\}
$$

Equation (26) is called the price of the geometric average Asian call option. The price of the geometric Asian put option can be obtained using put-call parity.

Equation (26) shows that Black-Scholes framework can 
be used for the valuation of geometric average Asian options.
The value of Geometric Asian options denoted by $f\left(t, S_{t}, G\right)$ satisfies the partial differential equation given by

\section{Mellin Transform for the Valuation of Geometric Asian Option}

$$
\left.\begin{array}{r}
\frac{\partial f\left(t, S_{t}, G\right)}{\partial t}+\frac{1}{2} \sigma^{2} S_{t}^{2} \frac{\partial^{2} f\left(t, S_{t}, G\right)}{\partial S_{t}^{2}}+r S_{t} \frac{\partial f\left(t, S_{t}, G\right)}{\partial S_{t}}+S_{t} \frac{\partial f\left(t, S_{t}, G\right)}{\partial G}=r f\left(t, S_{t}, G\right) \\
f\left(T, S_{t}, G\right)=\theta\left(S_{t}, G\right)
\end{array}\right\}
$$

Where $S_{t}$ is the underlying price of the asset, $r$ is the risk-neutral interest rate, $\sigma$ is the volatility, $T$ is the maturity date and $G=e^{\frac{1}{t} \int_{0}^{t} \ln S_{t} d t \text { is the average of the stock }}$ price at time $t$. The payoffs function for geometric Asian options are (i) floating strike call:

$\theta\left(S_{t}, G\right)=\max \left(S_{t}-G, 0\right)$ (ii) floating strike put:

$\theta\left(S_{t}, G\right)=\max \left(G-S_{t}, 0\right)$ (iii) fixed strike call:

$\theta\left(S_{t}, G\right)=\max (G-K, 0)$ (iv) fixed strike put:

$$
\theta\left(S_{t}, G\right)=\max (K-G, 0)
$$

The Mellin Transform for geometric Asian option, $A_{G}$ is given by

$$
\widehat{A}_{G}(w, t)=\int_{0}^{\infty} A_{G}\left(S_{t}, t\right) S^{w-1} d S
$$

Where $w$ is a complex variable with $0<\operatorname{Re}(w)<\infty$. The inversion of the Mellin transform is also given by

$$
\left.\begin{array}{r}
A_{p}\left(S_{t}, t\right)=\frac{1}{2 \pi i} \int_{c-i \infty}^{c+i \infty} \hat{A}_{p}(w, t) S^{-w} d w \quad \\
M\left(\frac{\partial f\left(t, S_{t}, G\right)}{\partial t}+\frac{1}{2} \sigma^{2} S_{t}^{2} \frac{\partial^{2} f\left(t, S_{t}, G\right)}{\partial S_{t}^{2}}+r S_{t} \frac{\partial f\left(t, S_{t}, G\right)}{\partial S_{t}}+S_{t} \frac{\partial f\left(t, S_{t}, G\right)}{\partial G}\right)=M\left(r f\left(t, S_{t}, G\right)\right) \\
M\left(f\left(T, S_{t}, G\right)\right)=M\left(\theta\left(S_{t}, G\right)\right)
\end{array}\right\}
$$

Therefore

$$
\left.\begin{array}{r}
\frac{\partial \hat{f}(t, w, G)}{\partial t}+\frac{1}{2} \sigma^{2}\left(w^{2}+w\right) \hat{f}(t, w, G)+r w \hat{f}(t, w, G)+\frac{\partial \hat{f}(t, 1+w, G)}{\partial G}=r \hat{f}(t, w, G) \\
\hat{f}(T, w, G)=\theta\left(S_{t}, G\right)
\end{array}\right\}
$$

Rearranging (34) we have that

$$
\left.\begin{array}{rl}
\frac{\partial \hat{f}(t, w, G)}{\partial t}+\frac{\partial \hat{f}(t, 1+w, G)}{\partial G}+\frac{1}{2}\left(\sigma^{2}\left(w^{2}+w\right)-r w-r\right) \hat{f}(t, w, G) & =0 \\
\hat{f}(T, w, G) & =\theta\left(S_{t}, G\right)
\end{array}\right\}
$$


But $\hat{f}(T, w, G)=\theta\left(S_{t}, G\right)$ can also be written for floating call and put respectively as

$$
\left.\begin{array}{l}
\hat{f}(T, w, G)=\max \left(-\frac{S_{0}^{1+w}}{1+w}-G, 0\right) \\
\hat{f}(T, w, G)=\max \left(G-\left(-\frac{S_{0}^{1+w}}{1+w}\right), 0\right)
\end{array}\right\}
$$

By means of change of variables, the first part of (35) becomes

$$
\tilde{f}(t, w, G)=g(t, w, G) e^{\left[\frac{1}{2}\left(\sigma^{2}\left(w^{2}+w\right)-r w-r\right)\right] \tau}, \tau=T-t
$$

Also

$$
\frac{\partial g(t, w, G)}{\partial \tau}=\frac{\partial g(t, 1+w, G)}{\partial G}
$$

Using Fourier transforms in $w$ and then we have

$$
\frac{\partial \tilde{g}(t, \tilde{w}, G)}{\partial \tau}=e^{i w} \frac{\partial \tilde{g}(t, \tilde{w}, G)}{\partial G}
$$

Solving (39) we have

$$
\tilde{f}(t, w, G)=\frac{\theta(w, G)}{G e^{i w}}((T-t) \Delta(\tilde{w})+G \Delta(\tilde{w}-1)) e^{\left[\frac{1}{2}\left(\sigma^{2}\left(w^{2}+w\right)-r w-r\right)\right](T-t)}
$$

Since

$f(t, w, G)=M^{-1}(\tilde{f}(t, w, G))$

Therefore,

$$
\left.f(t, w, G)=\frac{1}{2 \pi} \int_{\alpha-i \infty}^{\alpha+i \infty} S^{-w} \frac{\theta(w, G)}{G e^{i w}}((T-t) \Delta(\tilde{w})+G \Delta(\tilde{w}-1)) e^{\left[\frac{1}{2}\left(\sigma^{2}\left(w^{2}+w\right)-r w-r\right)\right](T-t)} d w\right\}
$$

Equation (45) is the analytic solution for geometric Asian options via the Mellin transform method.

\section{Conclusion}

In this paper we considered the Mellin transform method as an alternative analytical solution for the valuation of geometric Asian option. We also derived the closed form solution for geometric Asian option in the Black-Scholes framework. The Mellin transform method is also a powerful technique for the valuation of more complex vanilla options and some path dependent options and can be extended to jump diffusion processes, stochastic volatility and other stochastic processes.

2010 Mathematics Subject Classification: 11A25, 44A10, 60H30, 91G20, $91 \mathrm{G} 80$

\section{References}

[1] Fadugba S.E., Adefolaju F.H. and Akindutire O.H. (2012), On the stability and accuracy of finite difference method for options pricing, Mathematical Theory and Modeling, Vol. 2, No. 6, pp. 101-108.

[2] Fadugba S.E., Okunlola J.T. and Adeyemo A.O. (2013), On the robustness of binomial model and finite difference method for pricing European options, International Journal of IT, Engineering and Applied Sciences Research, Vol. 2, No. 2, pp. 5-11.

[3] Fadugba S.E. and Edogbanya O.H. (2014), On the valuation of credit risk via reduced-form approach, Global Journal of Science Frontier Research, Vol. 14, No. 1, Version 1, pp. 49-61. 
[4] Geman H. and Yor M. (1993), Bessel processes, Asian options and perpetuities, Mathematical Financial, Vol. 3, 349 -375, doi:10.1111/j.1467-9965.1993.tb00092.x.

[5] Lapeyre B. and Temam E. (2001), Competitive Monte Carlo methods for the pricing of Asian options, Journal of computational finance, Vol. 5, pp. 39-57.

[6] Nwozo C.R. and Fadugba S.E. (2012), Monte Carlo method for pricing some path dependent options, International Journal of Applied Mathematics, Vol. 25, No. 6, pp. 763-778.

[7] Nwozo C. R. and Fadugba S. E. (2012), Some numerical methods for options valuation, Communications in Mathematical Finance, Vol. 1, No. 1, pp. 51-74, United Kingdom

[8] Nwozo C.R. and Fadugba S.E. (2014), Performance measure of Laplace transforms for pricing path dependent options, International Journal of Pure and Applied Mathematics, Vol. 94, No. 2, pp. 175-197, dx.doi.org/10.12732/ijpam.v94i2.5.

[9] Panini R. and Srivastav R.P. (2004), Option Pricing with Mellin Transforms, Mathematical and Computer Modelling, Vol. 40, pp. 43-56, doi:10.1016/j.mcm.2004.07.008.

[10] Rogers L.C.G. and Shi Z. (1992), The value of an Asian option, Journal of Applied Probability, Vol. 32 pp. 10771088, doi:10.2307/3215221.

[11] Temam E. (2001), Couverture Approche des options exotiques. Pricing des options Asiatiques Ph.D. Thesis\}, University of Paris VI.

[12] Turnbull S.M. and Wakeman L.M.(1991), A quick algorithm for pricing European average options, Journal of Financial and Quantitative Analysis, Vol. 26, pp. 377-389, doi: $10.2307 / 2331213$.

[13] Vasilieva O. (2009), A New Method of Pricing MultiOptions using Mellin Transforms and Integral Equations, Master's Thesis in Financial Mathematics, School of Information Science, Computer and Electrical Engineering, Halmsta University.

[14] Yakubovich S.B. and Nguyen T.H. (1991), The Double Mellin-Barnes Type Integrals and their Applications to Convolution Theory, Series on Soviet Mathematics, World Scientific, 199.

[15] Yang Z., Oliver C.O. and Menkens O. (2011), Pricing and hedging of Asian options: quasi-explicit solutions via Malliavin calculus, Math Meth Oper Res, 74 pp. 93-120, doi:10.1007/s00186-011-0352-7.

[16] Zieneb A.E. and Rokiah R.A. (2011), Analytical Solution for an Arithmetic Asian Option using Mellin Transforms, Vol. 5, pp. 1259-1265

[17] Zieneb A.E. and Rokiah R.A. (2013), Solving an Asian options PDE via Laplace transform, ScienceAsia, 39S, pp. 67-69. 